\title{
ARTÍCULO ORIGINAL \\ Uso de quelonios continentales en el golfo de Morrosquillo, Sucre, Colombia
}

\author{
Freshwater turtle use on the Morrosquillo Gulf, Sucre, Colombia
}

ARDILA-MARULANDA, MERLY ${ }^{1} s p$, DE LA OSSA V, JAIME ${ }^{2}$ Ph.D, DE LAOSSA-LACAYO, ALEJANDRO ${ }^{3}$ M.SC.

\begin{abstract}
${ }^{1}$ Universidad de Sucre - SUE Caribe. Maestría en Ciencias Ambientales, Grupo de Investigación en Biodiversidad Tropical. Sucre, Colombia. ${ }^{2}$ Universidad de Sucre, Grupo de Investigación en Biodiversidad Tropical, Colombia. ${ }^{3}$ Universidad de Sucre. Grupo de Investigación en Biodiverisidad Tropical, Colombia. Selvagua SAS.
\end{abstract}

\section{Key words:}

Freshwater turtle; cattle ponds; utilization; Caribbean; Colombia.

\section{Abstract}

At Morrosquillo gulf, in Sucre, Colombia it is possible to find many waterholes which are occupied by freshwater turtles of which were selected 65 with an area greater than $1,000 \mathrm{~m}^{2}$; a representative sample of 12 randomly selected cattle ponds that were not linked was used. The population data were collected with samples taken between January and March of 2014. Interactive dialogues were held with members of the community in order to identify people who are engaged in hunting, trading or consuming turtles of economic interest; surveys were carried out to gather information on the availability and use of captured turtles. The users of this resource were all male with $30 \%$ illiteracy, opportunistic exploiters of the turtles that obtain an annual income of between $\$ 300,000.00$ and $\$ 400,000.00$ Colombian Pesos. The most widely used turtle was $T$. callirostris, which was sold or consumed by the families.

\section{Resumen}

En el Golfo de Morrosquillo, Sucre, Colombia, se encuentran un gran número de jagüeyes que son ocupados por quelonios continentales, de los cuales se seleccionaron 65 con área mayor a $1.000 \mathrm{~m}^{2}$, se trabajó con una muestra de 12 jagüeyes escogidos al azar y sin comunicación alguna entre ellos. Los muestreos se realizaron entre enero y marzo de 2014. En la comunidad se llevaron a cabo diálogos interactivos para identificar a los pobladores que se dedican a la cacería, comercio o consumo de quelonios de interés económico; se les aplicaron encuestas para recopilar la información sobre la disponibilidad y patrones de uso de los quelonios capturados. Los usuarios del recurso son en su totalidad hombres con un $30 \%$ de analfabetismo, realizan aprovechamiento oportunista de los quelonios y derivan ingresos anuales entre Col. \$300.000. oo y 400.000.oo. El quelonio de mayor uso es T. callirostris, cuyos ejemplares son vendidos vivos o consumidos en el entorno familiar. 


\section{Introducción}

Colombia posee aproximadamente $10 \%$ de fauna y flora existente en el planeta, lo cual lo sitúa como uno de los más ricos en diversidad biológica; al igual que ocupa el cuarto puesto en número de reptiles (SÁNCHEZ et al., 1995), uno de los problemas que ha sido determinante en las dinámicas de la fauna y flora es el cambio climático, para hacerle frente es necesario preservar ecosistemas antiguos, y enfrentar la conformación generalizada de ecosistemas nuevos, que permitan conservar las especies, además de la amenaza del cambio climático, algunas especies de quelonios como Trachemys callirostris, Kinosternon scorpiodes y Batrachemys dahli, enfrentan otras amenazas como cacería ilegal, pérdida de hábitat y construcción de hidroeléctricas (MEDEM, 1956; 1966). Las leyes vigentes no han logrado controlar el uso de tales recursos y la práctica ilegal de su captura y comercio que están deteriorando el hábitat y colocando en peligro estas y otras especies (CONVENIO SENA-CORPOMOJANA, 1999).

Según de DE LA OSSA et al. (2011) en Colombia, país megadiverso, un grupo muy representativo son los reptiles entre estos se encuentran los quelonios de algunos de ellos se aprovecha la carne y los huevos como alimento y la concha o caparazón de casi todos se utilizan para elaborar artesanías, bisutería y fracciones de instrumentos musicales, lo que los convierte en especies importantes para las comunidades rurales e indígenas en áreas remotas (MORALES et al., 2013).

El bajo nivel cultural de la población del Golfo de Morrosquillo, es determinante en que los recursos naturales sean utilizados como medio de subsistencia ya que la población no cuenta con puestos de trabajo bien remunerados; ante este desequilibrio no se puede hablar de un verdadero desarrollo sostenible de los recursos naturales, ni de que la conservación de los mismos se pueda realizar aplicando métodos tradicionales ya que, no solo se trata de defender a la naturaleza del impacto del desarrollo, sino también de procurar que satisfaga las necesidades de recursos biológicos que tienen las poblaciones humanas (DE LA OSSA y SAMPEDRO, 1999).
Los quelonios desempeñan un papel muy importante para la comunidad, ya que se pudo establecer que son consumidos y comercializados, especialmente la hicotea, T. callirostris, sin embargo la comunidad señala que se ha presentado una disminución de la especie en la zona, ocasionados por el cambio climático y la sobreexplotación.

Es de anotar, que el estudio resulta pertinente para el Golfo de Morrosquillo en Sucre, ya que en esta zona existen varios jagüeyes de diferente dimensiones, donde persiste una relación positiva entre el número de jagüeyes y la biodiversidad local de un área dada (BOTERO et al., 2009), y puede brindar una información actualizada sobre los patrones de uso de los quelonios de la zona; además los resultados podrían ser útiles para determinar la situación actual de ese recurso faunístico

\section{Materiales y métodos}

Zona de estudio: La ubicación inicial del área de estudio y todos los jagüeyes existentes, así como las medidas para calcular su área y su ubicación se hicieron mediante la aplicación del programa Google Earth (versión libre 7.1.2.2041), con imagen reciente 07-2014-2014. Luego se llevaron a un formato Keyhole Markup Language $(\mathrm{kml})$; todos los polígonos se trasladaron al formato shape (.shp) para ser utilizados en el software ArcMap de ArcGIS licenciado a la Universidad de Sucre los jagüeyes no deben presentar comunicación acuática entre ellos, ni estacional ni permanente (DE LA OSSA y DE LA OSSA-LACAYO, 2013). Se seleccionaron todos los polígonos con área mayor a $1.000 \mathrm{~m}^{2}$ de espejo de agua, que no tuvieran comunicación entre ellos, dando como resultado un total de 65, correspondiente a un área de 24,07 has.

En la Tabla 1 se relacionan los 12 sitios de muestreo, seleccionados aleatoriamente los cuales se localizan entre los municipios de San Onofre y Santiago de Tolú, departamento de Sucre, Colombia. 
Tabla 1. Coordenadas de cada lugar de muestreo y medidas de área para cada jagüey.

\begin{tabular}{cccc}
\hline Jagüey & Área $\mathrm{m}^{2}$ & Longitud & Latitud \\
\hline 1 & $6.545,80$ & $75^{\circ} 32^{\prime} 20^{\prime \prime}$ & $9^{\circ} 33^{\prime} 0^{\prime \prime}$ \\
2 & $1.190,00$ & $75^{\circ} 32^{\prime} 31^{\prime \prime}$ & $9^{\circ} 33^{\prime} 47^{\prime \prime}$ \\
3 & $32.509,85$ & $75^{\circ} 33^{\prime} 29^{\prime \prime}$ & $9^{\circ} 43^{\prime} 12^{\prime \prime}$ \\
4 & $6.814,26$ & $75^{\circ} 35^{\prime} 10^{\prime \prime}$ & $9^{\circ} 39^{\prime} 58^{\prime \prime}$ \\
5 & $8.796,13$ & $75^{\circ} 30^{\prime} 22^{\prime \prime}$ & $9^{\circ} 36^{\prime} 14^{\prime \prime}$ \\
6 & $3.185,12$ & $75^{\circ} 31^{\prime} 37^{\prime \prime}$ & $9^{\circ} 37^{\prime} 48^{\prime \prime}$ \\
7 & $3.174,95$ & $75^{\circ} 35^{\prime} 35^{\prime \prime}$ & $9^{\circ} 41^{\prime} 24^{\prime \prime}$ \\
8 & $25.652,74$ & $75^{\circ} 31^{\prime} 41^{\prime \prime}$ & $9^{\circ} 43^{\prime} 30^{\prime \prime}$ \\
9 & $4.083,78$ & $75^{\circ} 33^{\prime} 7^{\circ}$ & $9^{\circ} 38^{\prime} 31^{\prime \prime}$ \\
10 & $1.172,37$ & $75^{\circ} 32^{\prime} 2^{\prime \prime}$ & $9^{\circ} 41^{\prime} 10^{\prime \prime}$ \\
11 & $1.388,17$ & $75^{\circ} 35^{\prime} 56^{\prime \prime}$ & $9^{\circ} 43^{\prime} 8^{\prime \prime}$ \\
12 & $2.831,50$ & $75^{\circ} 35^{\prime} 10^{\prime \prime}$ & $9^{\circ} 42^{\prime} 0^{\prime \prime}$ \\
\hline Área Total & $97.344,67$ & & \\
\hline
\end{tabular}

La toma de información poblacional se realizó mediante muestreos llevados a cabo entre enero y marzo de 2014, entre las 08:00 y las 12:00 horas, utilizando un trasmallo $100 \mathrm{~m}$ de largo por $6 \mathrm{~m}$ de ancho y ojo de $5 \mathrm{~cm}$ y en cada sesión se hicieron dos barridos; manteniendo un cubrimiento del $90 \%$ en los arrastres del área de cada jagüey.

Mediante encuestas semiestructuradas, según lo establecido por MARGOLUIS y SALAFSKY (1998), se colectó la información socioeconómica, perceptiva y de utilización del recurso, para lo cual se tuvo en cuenta un proceso de dos niveles.

Inicialmente se entablaron diálogos interactivos con miembros de la comunidad que habita la zona de estudio, para identificar a las personas que se dedican a la cacería, comercio y/o consumo de quelonios, con esta metodología participativa e informal se obtendrá cualitativamente información sobre el uso del recurso y la percepción del mismo.

Se aplicaron encuestas semiestructuradas, siguiendo las consideraciones descritas para recopilar información ecológica sobre la disponibilidad y el uso de las especies encontradas. Durante el estudio, se trabajó con una muestra representativa equivalente al $50 \%$ de los potenciales cazadores, que se identificaron en los diálogos previos. Una vez recolectada la información los datos de captura fueron integrados a una base de datos, procesados para el análisis descriptivo y posteriormente llevada al paquete estadístico SPSS versión free (Licencia Pública).

El análisis de datos cuantitativos de encuestas y registros de captura, fue analizado mediante la aplicación y representación de tablas. Para comparar las muestras se utilizaron cálculos de porcentajes

\section{Resultados}

Existe diferencia significativa entre jagüeyes al comparar sus áreas mediante prueba de t simple (Std. Dv $=10187,09 . \mathrm{df}=11, \mathrm{~T}=2,758330, \mathrm{p}=$ $0,018611)$. Existe una diferencia significativa en todas las especies, entre los inmaduros, machos y hembras (Tabla 2).

Tabla 2. Captura por especie, sexo y estado de madurez.

\begin{tabular}{ccccc}
\hline Especie & Inmaduro & Macho & Hembra & Total especie \\
\hline Trachemys callirostris & 58 & 43 & 140 & 241 \\
Kinosternon scorpiodes & 7 & 18 & 41 & 66 \\
Batrachemys dahli & 0 & 23 & 53 & 76 \\
Total & $\mathbf{6 5}$ & $\mathbf{8 4}$ & $\mathbf{2 3 4}$ & $\mathbf{3 8 3}$ \\
\hline
\end{tabular}

$X^{2}=28.020^{\text {a Significancia }=000}$

En la Tabla 3 se presenta el aprovechamiento del recurso que es efectuado totalmente por personas del género masculino los cuales han sido distribuidos según sus edades

Tabla 3. Distribución de edades en los encuestados en la zona de estudio.

\begin{tabular}{ccccc}
\hline Rango de edad & \multicolumn{2}{c}{ Cazadores encuestados en 3 distintas zonas } & Total \\
\hline $20-30$ & 4 & 2 & 1 & 7 \\
$31-40$ & 2 & 7 & 2 & 11 \\
$41-50$ & 0 & 1 & 1 & 2 \\
Total & $\mathbf{6}$ & $\mathbf{1 0}$ & $\mathbf{4}$ & $\mathbf{2 0}$ \\
\hline
\end{tabular}

De los entrevistados se tiene un $30 \%(n=6)$ de analfabetismo. Se tiene una media de 3,8 hijos/ familia. En la Tabla 4 se presenta número de hijos por familia y nivel máximo de escolaridad de al menos uno de sus miembros.

Tabla 4. Número de hijos y grado de escolaridad

\begin{tabular}{cccc}
\hline $\begin{array}{c}\text { Número de } \\
\text { familias }\end{array}$ & $\begin{array}{c}\text { Número medio de } \\
\text { hijos/familia }\end{array}$ & $\begin{array}{c}\text { Número medio del } \\
\text { grupo familiar }\end{array}$ & $\begin{array}{c}\text { Escolaridad } \\
\text { máxima }\end{array}$ \\
\hline 3 & 2 & 4 & Primaria \\
13 & 3 & 5 & Primaria \\
2 & 4 & 6 & Secundaria \\
2 & 5 & 7 & Primaria \\
\hline
\end{tabular}


En la Tabla 5 se relacionan los ingresos mensuales que perciben se ubican cerca de un salario mínimo mensual legal vigente, no se detectaron ingresos familiares adicionales.

Tabla 5. Ingresos económicos generados por empleo.

\begin{tabular}{cccc}
\hline Actividad & $\mathbf{n}$ & Ingreso (\$ 000) & Ingreso familiar (\$ 000) \\
\hline Trabajador ganadería & 14 & 650 & 650 \\
Jornalero & 6 & 550 & 550 \\
\hline Media & & $\mathbf{6 0 0}$ & $\mathbf{6 0 0}$ \\
\hline
\end{tabular}

No registraron vivienda propia, ocupan casas de las fincas en las que laboran y no poseen tierras. El único servicio público con que cuentan todos es luz eléctrica; el servicio de salud en un $70 \%$ $(n=14)$ es dado por el SISBEN, 70\% $(n=14)$ de los encuestados cocina con leña, el otro $30 \%(n=6)$ con gas propano. Los demás servicios públicos se discriminan en la Tabla 6.

Tabla 6. Servicios públicos que poseen los entrevistados.

\begin{tabular}{ccc}
\hline Servicio público & $\mathbf{n}$ & $\%$ \\
\hline Agua potable & 5 & 25 \\
Letrina & 7 & 35 \\
\hline
\end{tabular}

Los encuestados coincidieron en afirmar que la cachama (Colossoma macropomum) es la especie de cultivo más consumida por los quelonios (Tabla 7).

Tabla 7. Especies de peces de cultivo que son consumidas por los quelonios.

\begin{tabular}{cccc}
\hline Especie & N. común & $\mathbf{n}$ & $\%$ \\
\hline Prochilodus madgdalenae & Boachico & 15 & 75 \\
Colossoma macropomum & Cachama & 18 & 90 \\
Tarpon atlanticus & Sábalo & 15 & 75 \\
Tilapia spp & Tilapia & 10 & 50 \\
\hline
\end{tabular}

Respecto de los beneficios que se obtiene de los quelonios, el total de los encuestados consideran que son un alimento alternativo cuando se presenta escasez de proteína proveniente de otras especies; además, porcentualmente le atribuyen valor absoluto al mantenimiento del agua en los jagüeyes y consideran benéfico que ejerzan un control sobre las poblaciones de serpientes y anfibios ya que éstos son considerados predadores de los alevinos que cultivan en los jagüeyes y en un menor porcentaje son considerados fuente de ingresos al ser comercializados vivos en los mercados locales (Tabla 8).
Tabla 8. Beneficios que obtienen los encuestados de los quelonios.

\begin{tabular}{ccc}
\hline Beneficios & $\mathbf{n}$ & $\%$ \\
\hline Son alimento & 20 & 100 \\
Mantienen limpia el agua & 20 & 100 \\
Consumen serpientes & 4 & 20 \\
Consumen anfibios & 16 & 80 \\
Ingresos adicionales & 7 & 35 \\
\hline
\end{tabular}

De los especies capturadas se utilizan el caparazón y la carne, la primera para la elaboración de artesanías y la segunda para autoconsumo, solo se comercializa la especie $T$. callirostris viva, de forma ilegal por lo que no existen registros de esta actividad. El consumo de cada especie expresado porcentualmente se presenta en la Tabla 9).

Tabla 9. Consumo de las especies por parte de los encuestados.

\begin{tabular}{ccc}
\hline Especie & $\mathbf{N}^{\circ}$ & $\%$ \\
\hline Trachemys callirostris & 18 & 90 \\
Kinosternon scorpiodes & 2 & 10 \\
Batrachemys dahli & 1 & 5 \\
\hline
\end{tabular}

Los veinte encuestados manifiestan que utilizan el arrastre y el chuzo para la caza de quelonios, pero que no tienen datos concretos sobre el número de individuos capturados y calculan que el ingreso generado por esta actividad puede oscilar entre $\$ 350.000 .00$ a $\$ 500.000 .00$ anual per cápita

\section{Discusión}

Ante los fuertes cambios climáticos y las sequias que se presentan en el golfo de Morrosquillo, Sucre, se tienen los jagüeyes que son cuerpos de agua construidos, por las comunidades para el suministro de agua, o por los ganaderos como abrevadero, son sin duda, un elemento importante en la conservación local de quelonios continentales: T. callirostris, $K$. scorpiodes y $B$. dahli. Aspecto que coincide con lo señalado para este tipo de unidades de almacenamiento de agua por BOTERO et al. (2009). Todos los cuerpos de agua, independientemente de su tamaño, juegan un papel muy importante en la preservación de los quelonios, especialmente si factores antrópicos como el deterioro de hábitat limitan la capacidad de carga de los ecosistemas y hacen que 
estrategias como la colonización sean valiosas para la sobrevivencia (DE LA OSSA, 1996).

No existe diferencia significativa entre el tamaño del jagüey y el número de individuos capturados por especie, lo que indica que todos los cuerpos de agua, independientemente de su tamaño, juegan un papel muy importante en la preservación de los quelonios, Especies de quelonios dulce acuícolas como $T$. callirostris, $K$. scorpiodes y $B$. dahli, a pesar de la degradación de su entorno natural se han mantenido en la zona de estudio gracias a la existencia de los jagüeyes que les ofrecen condiciones acordes con sus necesidades vitales.

Las poblaciones estimadas para cada una de las especies de quelonios continentales de este trabajo, se encuentran distribuidas en los 12 jagüeyes, independientemente del tamaño del mismo. Aunque la población estimada para cada especie y jagüey, no es abundante, en comparación con los resultados obtenidos para otros quelonios de agua dulce que frecuentan principalmente cuerpos de agua de mayor dimensión, cómo Trachemys scripta en Isla Coco de La Mojana (FUENTES-OBEID et al., 2003), si se convierte en un punto de partida para el uso de los ecosistemas antrópicos como alternativa de conservación de los quelonios de agua dulce. Sin embargo, se debe prestar especial atención, ya que por su localización y uso agrícola, es factible que haya contaminación en estos cuerpos de agua, producto de los diversos usos que le da la comunidad a los jagüeyes, unido a la escorrentía de los productos químicos utilizados en los cultivos cercanos (CASTAÑO et al., 2005).

Sucre ubica su nivel de analfabetismo general en un poco más del $7 \%$ y ocupa uno de los últimos lugares, con valor muy similar a Magdalena, Cesar, Vichada y Córdoba, por debajo de Guajira y Chocó (RODRÍGUEZ-TORRES, 2012). En índice de analfabetismo detectado para la muestra de personas con que se trabajó en el presente estudio hace referencia a población rural, la cual generalmente está por encima de la que se localiza a nivel urbano, es significativamente muy alta y cercana a la mayor tasa nacional de analfabetismo, que se estima que para Colombia es del 9,8 según informe del MINISTERIO DE EDUCACIÓN (2014).

En el estudio se registró que en la mayoría de las familias solo una persona participa en las actividades de caza y/o comercialización de fauna silvestre y generalmente es el padre, pero los ingresos obtenidos por esta actividad no son representativos. Durante la temporada de captura; según BAPTISTE et al. (2009), la actividad puede proveer ingresos estables equivalentes o cercanos a un salario mínimo mensual vigente a una familia dedicada a esta actividad, lo cual no se registra en este caso, ya que los cazadores realizan estas actividades esporádicamente y el ingreso es comparativamente menor.

El uso de quelonios puede establecerse como un patrón social de subsistencia, aun cuando legalmente la cacería de subsistencia esté referida tácitamente a la alimentación del usuario, según lo preceptuado en el Decreto 1608/1978, Art. 31, señala: "El aprovechamiento de la fauna silvestre y de sus productos sólo podrá adelantarse mediante permiso, autorización o licencia que se podrán obtener en la forma prevista por este Decreto". Agrega de forma puntual y única: "La caza de subsistencia no requiere permiso pero deberá practicarse en forma tal, que no se causen deterioros al recurso. La entidad administradora organizará sistemas para supervisar su ejercicio". No obstante, hay que resaltar, coincidiendo con DE LA OSSA y DE LA OSSA- LACAYO (2011). Sin embargo, se pudo constatar que el comercio de Trachemys callirostris es apetecido por razones culturales y subsistencia, seguido de Kinosternon scorpiodes y ante la ausencia de proteína algunas personas han iniciado el consumo de Batrachemys dahli, lo cual manifiesta un gradual efecto de substitución (DE LA OSSA y VOGT, 2010).

Es de suma importancia proteger los jagüeyes como unidad ecológica que garantice la sostenibilidad de la biodiversidad local, en esta actividad deben involucrarse los diferentes actores ambientales así como los propietarios de fincas o las empresas ganaderas, y los habitantes de la comunidad. 


\section{Referencias}

BAPTISTE, L.G.; HERNÁNDEZ, S.; POLANCO, R.; QUICENO, M.P. 2009. La fauna silvestre Colombiana: una historia económica y social de un proceso de marginalización. Disponible en: http://www.humboldt. org.co/pdf/usoyval/Baptiste.pdf. Consultado: 12-08-2016.

BOTERO, A.L.; DE LA OSSA. V.J.; ESPITIA. A.; DE LA OSSA-LACAYO, A., 2009. Importancia de los jagüeyes en las sabanas del Caribe colombiano. Rev. Colombiana Cienc. Anim. 1 (1):71-84.

CASTAÑO, O.; CÁRDENAS, G.; GALLEGO, N.; RIVERA, O. 2005. Protección y conservación de los quelonios continentales en el departamento de Córdoba. Corporación autónoma de los valles del Sinú y San Jorge-CVS. Montería, Colombia

CONVENIO SENA-CORPOMOJANA. 1999. Diseño e instrumentación de un modelo pedagógico y tecnológico para el manejo sostenible de los recursos hidrobiológicos y del ponche, Hidrochaeris hidrocaheris isthmius, en La Mojana y el San Jorge. Sucre, Colombia.

DE LA OSSA V.J.; SAMPEDRO, A. 1999. Una aproximación analítica sobre la necesidad de considerar la sostenibilidad de la fauna silvestre como una alternativa de desarrollo. Biología 13 (2):79 - 82

DE LA OSSA, V.J. 1996. Colonización y ocupación territorial de lagunas artificiales por Caiman crocodilus fuscus (Cope,1868) (Crocodylia Alligatoridae). Crocodiles. Págs. 117-130. En: Proccedind of the 13 Working Meeting of de Crocodile Specialists Group. The World Conservation Union. Gland, Switzertland.

DE LA OSSA, V.J.; DE LA OSSA-LACAYO, A. 2011. Cacería de subsistencia en San Marcos, Sucre, Colombia. Recia 3(2):213-224.

DE LA OSSA, V.J.; DE LA OSSA-LACAYO, A. 2013. Ocupación de jagüeyes por la babilla, Caiman crocodilus fuscus (Cope, 1868), en el Caribe colombiano. Biota colombiana 14 (2): 327-336.

DE LA OSSA, V.J.; OLIVERO, G.G.; RUIZ, J.G. 2011. Utilización de quelonios de interés económico en el Municipio de Caimito, Sucre, Colombia Rev. Colombiana cienc. Anim. 3 (1):3-14.

DE LA OSSA, V.J.; VOGT, R.C. 2010. Efecto de sustitución: una expresión del agotamiento poblacional de quelonios en Barcelos, Amazonas, Brasil. Rev. Asoc. Col. Cienc.(Col.), 22: 61-67.

FUENTES-OBEID, S.; SAMPEDRO, M.A.; ARDILA-MARULANDA, M. 2003. Importancia de la jicotea (Trachemys scripta callirostris: Chelonia, Emydidae) como recurso natural en la comunidad de isla del Coco, Región de La Mojana, Departamento de Sucre, Colombia. Revista Biología 17(2):126-133.

MARGOLUIS, R.; SALAFSKY, N. 1998. Measures of success: designing, managing, and monitoring conservation and development projects. Island Press. Washington, DC.

MEDEM, F. 1956. Informe sobre reptiles colombianos I. Noticias). Bogotá, Colombia.

MEDEM, F. 1966. Contribuciones al conocimiento sobre la ecología y distribución geográfica de Phrynops (Batrachemys) dahli ; (Testudinata, Pleurodira, Chelidae). Caldasia 9 (3):467-482.

MINISTERIO DE EDUCACIÓN (2014). Educación. Disponible en: http://www.mineducacion.gov.co/ cvn/1665/articles-101270_archivo_pdf1.pdf. Consultado: 21-09-2016. 
MORALES-BETANCOURT, M.A.; LASSO, CA.; DE LA OSSA, V.J.; FAJARDO-PATIÑO, A. 2013. VIII. Biología y conservación de los Crocodylia de Colombia. Serie Editorial Recursos Hidrobiológicos y Pesqueros Continentales de Colombia. Instituto de Investigación de Recursos Biológicos Alexander von Humboldt (IAvH). Bogotá, D. C., Colombia.

RODRÍGUEZ-TORRES, M.C. 2012. Educación y Pobreza, Un Análisis De Eficiencia Relativa Departamental. Universidad Nacional de Colombia, Facultad de Economía, Maestría en Ciencias Económicas. Bogotá, Colombia.

SÁNCHEZ, H; CASTAÑO, O.; CÁRDENA, G. 1995. Diversidad de los reptiles en Colombia. Págs. $227-$ 326. En: Rangel, O (ed.) Colombia Diversidad Biótica I. Convenio Inderena - Universidad Nacional de Colombia. Bogotá DC. 